\title{
Nannizzia incurvata as rare cause of favus and tinea corporis in
}

\section{Cambodia and Vietnam}

Silke Uhrlaß¹, Mey Sithach², Stefanie Storch ${ }^{3}$, Franziska Wittig ${ }^{1}$, Daniela Koch ${ }^{1}$, Constanze Krüger ${ }^{1}$, Pietro Nenoff ${ }^{1}$

${ }^{1}$ Laboratory for Medical Microbiology, Mölbiser Hauptstraße 8, Mölbis, Germany; ${ }^{2}$ Department of Dermatology, Preah Kossamak Hospital, Phnom Penh, Cambodia ${ }^{3}$ Dermatological Office Dr. Stefanie Storch, Göltzschtalstr. 56, Auerbach, Germany

Objectives: Nannizzia (N.) incurvata (formerly Microsporum incurvatum) represents a geophilic dermatophyte which has been previously classified into the species complex of $N$. gypsea (formerly Microsporum gypseum). According to the new taxonomy of dermatophytes,

$N$. incurvata should be considered as an own species. Two patients with an infection caused by $N$. incurvata are presented in this study. Both strains were identified macroscopically by culture and by Sanger sequencing of the ITS region of the rDNA and the TEF1- $\alpha$ gene.

Patient 1 (lab. No. 213959/2017)

42 years old, Vietnamese, female, living in Saxony in Germany returned from a 2-week visit to her homeland Vietnam

suffered from a burning tinea corporis of the right buttock (Figure 1)

- family members were not affected N. incurvata (Figure 2) was cultured from skin scrapings of the centrifugal lesions

\section{treatment}

- clotrimazole + betamethasone dipropionate ointment or ciclopirox olamine cream - no improvement

- mometason furoate - exacerbation

- monotherapy twice daily with ciclopirox olamine cream for 4 weeks

$\rightarrow$ healing up

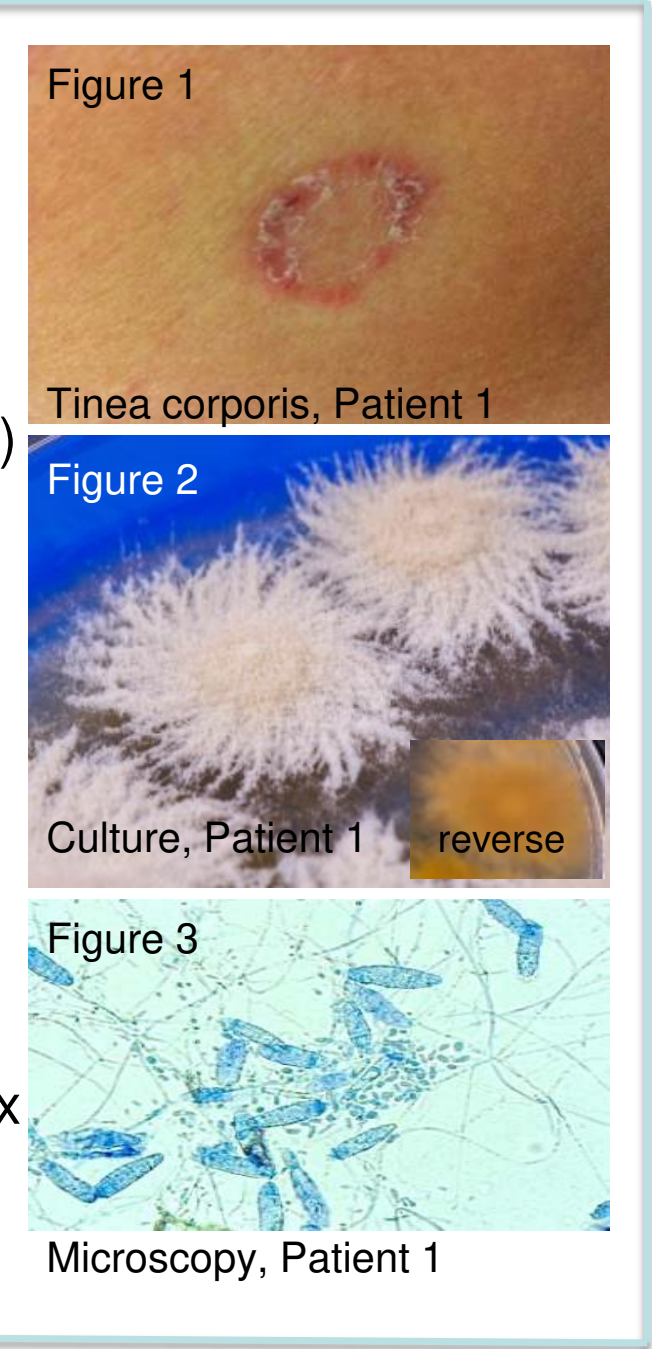

Mycological diagnostics

The strains were cultured on Sabouraud's dextrose agar and identified as $N$. incurvata by their macro-morphological (Figure 2, 5) and microscopic (Figure 3, 6) features.

Molecular biological diagnostics

Sequencing was used to confirm both strains to be $N$. incurvata.
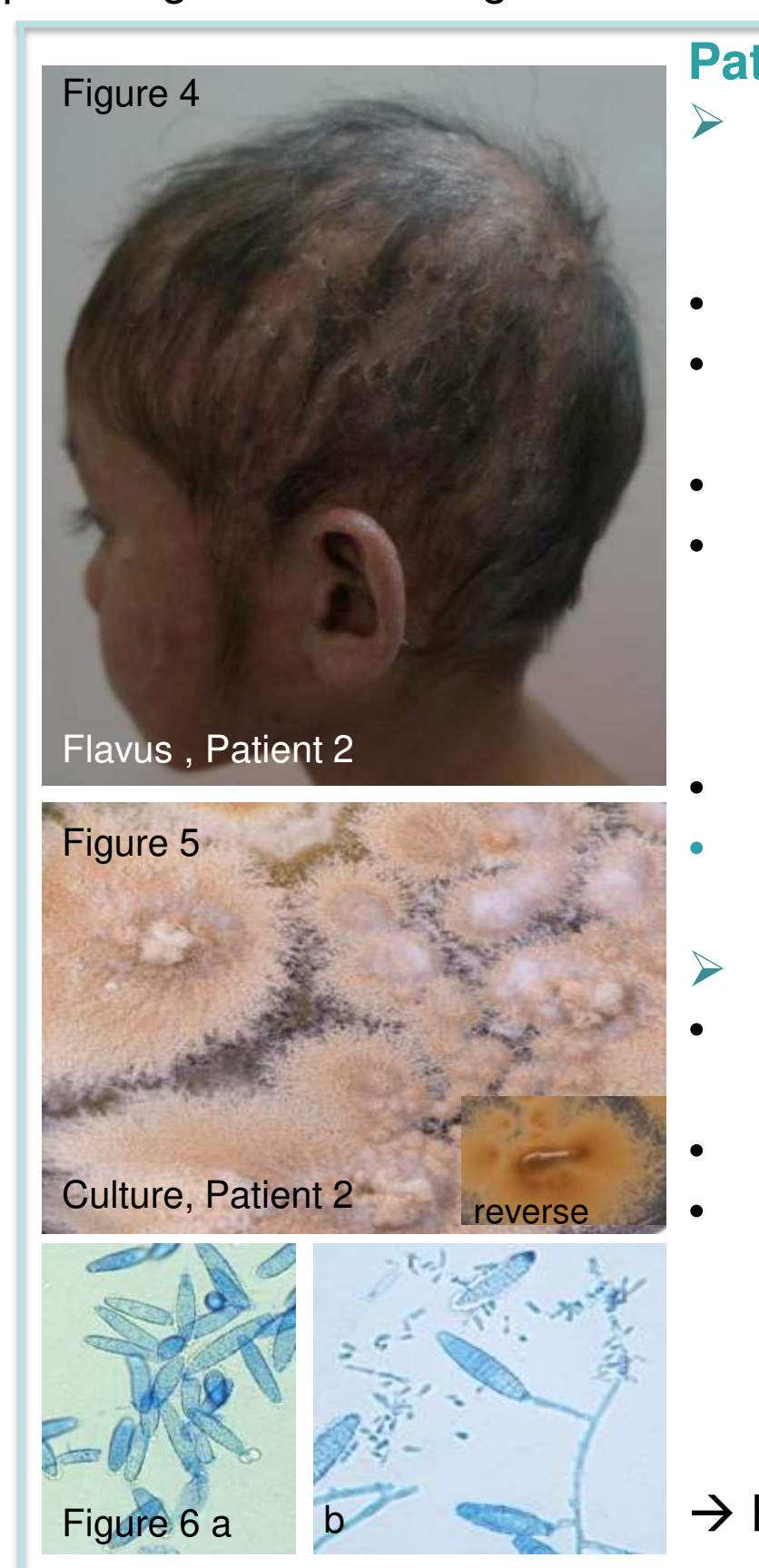

Microscopy, Patient 2

Patient 2 (lab. No. 211859/2017)

6 years old, Cambodian, male, living in a village near the river Mekong (water and forest region)

- contact to chicken, dogs, and cattle

suffered from tinea faciei and tinea capitis (Figure 4), symptoms for 2 years

family members were not affected initial lesion on the scalp, later extended to the face, favus- like tinea capitis and tinea faciei with erythema, scaly patches with areas of alopecia, but no pustules

main complaints were pruritus and alopecia N. incurvata (Figure 5) was cultured from skin scrapings

\section{treatment}

griseofulvin $250 \mathrm{mg}$ once daily for 12 weeks directed by a paediatrician - no improvement - Hepatitis B and C and HIV test - negative oral terbinafine $125 \mathrm{mg}$ daily, topical miconazole, ketoconazole shampoo, oral cetirizine, and multivitamine preparations for 2 weeks at the Department of Dermatology in Phnom Penh, Cambodia

$\rightarrow$ healing up after one month

ITS region (partial 18S rRNA, ITS1, 5.8S rRNA, ITS2, and partial 28S rRNA) and TEF1- $\alpha$ (Translation Elongation Factor 1- $\alpha$ )

- ITS region strain 213959/2017 - MF 415405.1 and strain 211859/2017 - MF415404.1 from NCBI

- TEF1 - $\alpha$ strain 213959/2017 - no cluster to compare and strain 211859/2017 - KM678105.1 from NCBI

Note: Due to the new taxonomy and nomenclature change KM678105.1 was assigned as N. gypsea.

strain 213959/2017, Patient 1, DSM106637 and strain 211859/2017, Patient 2, DSM106636 are deposited at DSMZ in Braunschweig, Germany

ITS region and TEF1- $\alpha$ sequences of both isolated strains will be deposited at the NCBI, the ISHAM ITS Database, and at the Fungal MLST Database

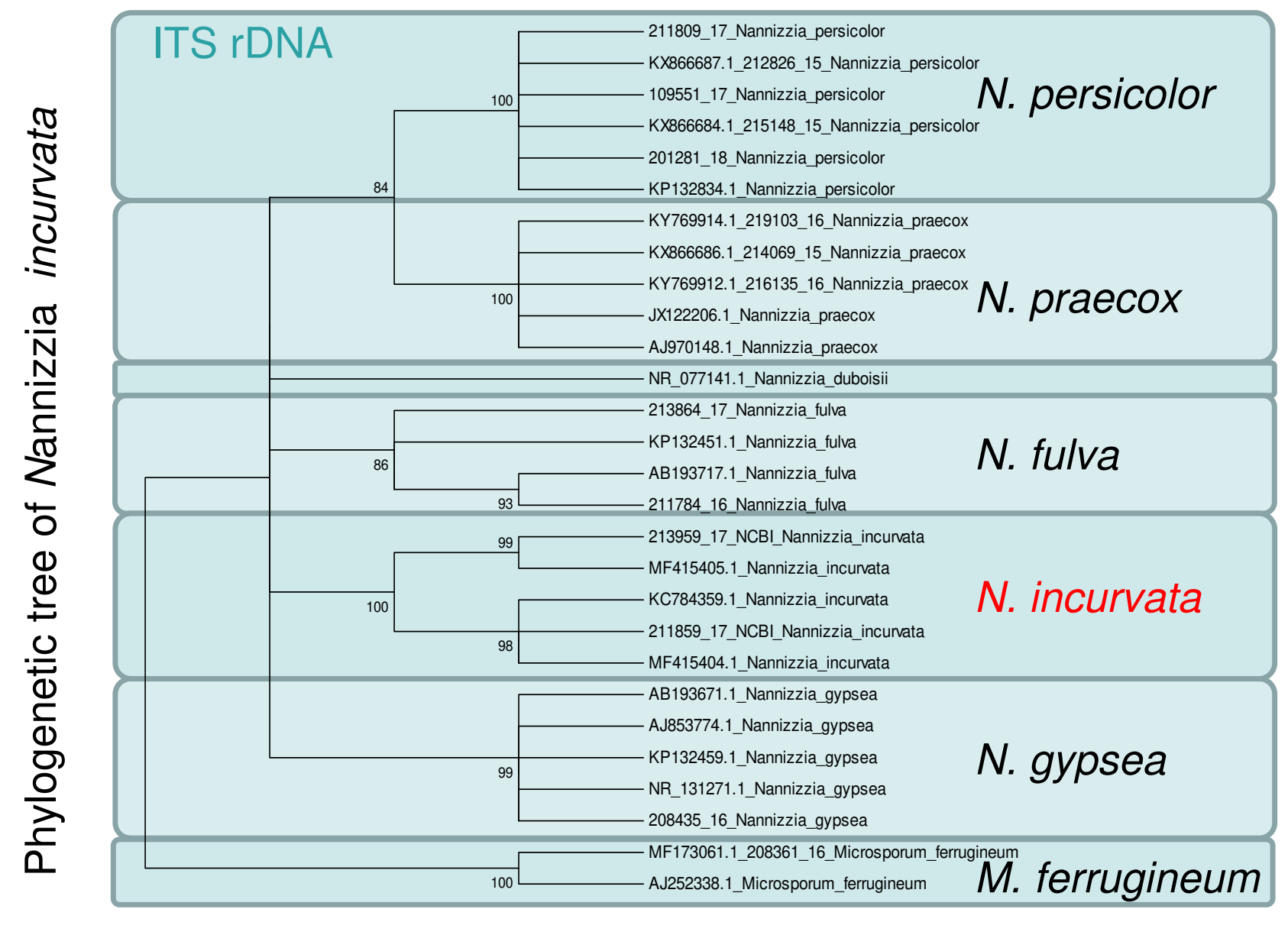

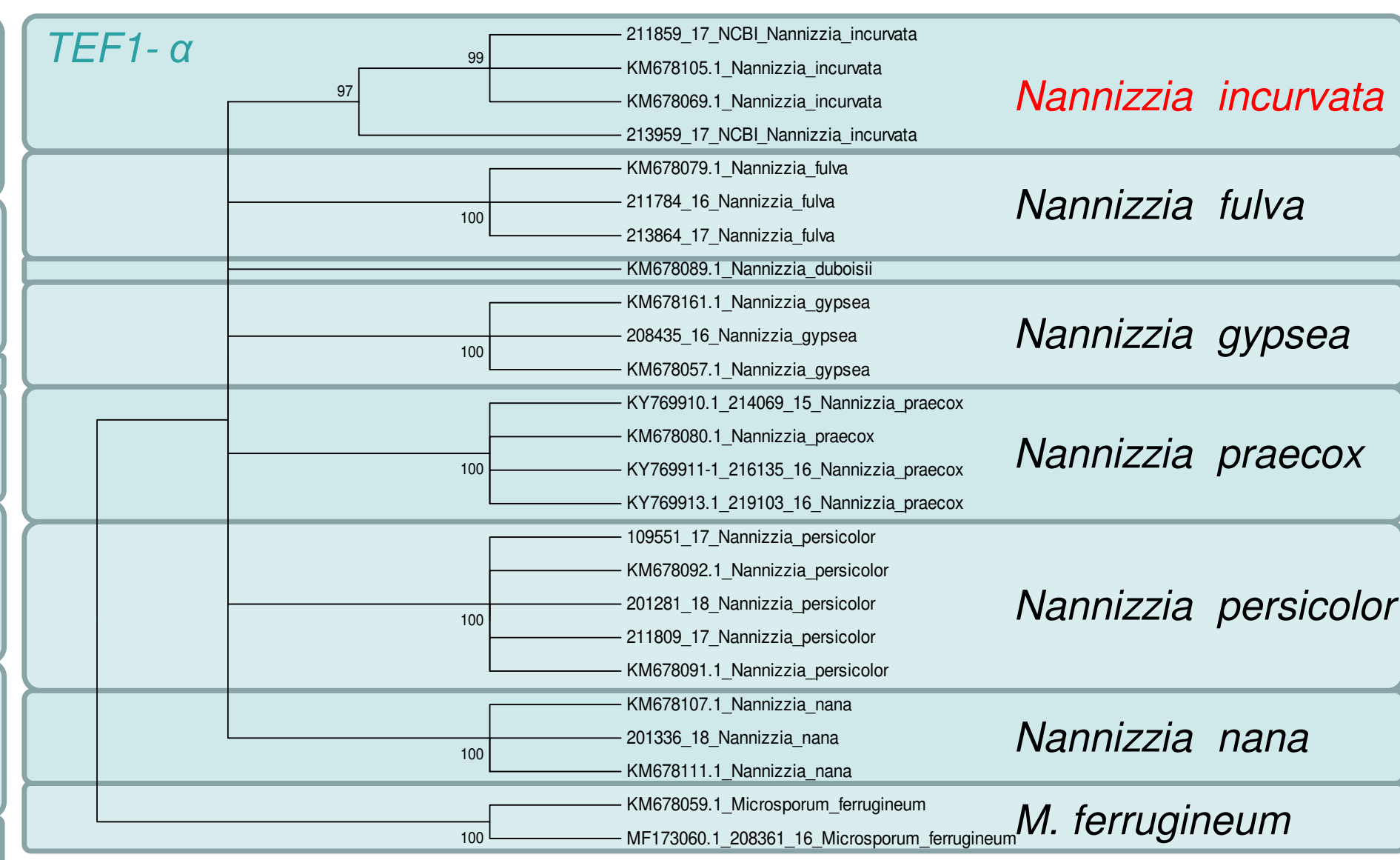

Note: Strains with letters...NCBI; Strains with numbers...routine samples from the lab

Generated by Mega 6.06, Statistical Method Maximum Likelihood, Bootstrap Replication 1000, rooted with Microsporum ferrugineum

Conclusion: $N$. incurvata is a rare geophilic dermatophyte first described in Sri Lanka and Vietnam. This study shows the first isolation of $N$. incurvata in Cambodia, and the first description of a favus in a child caused by this dermatophyte.

- de Hoog GS, Dukik K, Monod M, Packeu A, Stubbe D, Hendrickx M, Kupsch C, Stielow JB, Freeke, Göker M, Rezaei-Matehkolaei A, Mirhendi H, Gräser Y. Toward a novel multilocus phylogenetic taxonomy for the dermatophytes. Mycopathologia 2017; 182: 5-31 Do NA, Nguyen TD, Nguyen KL, Le TA. Distribution of species of dermatophyte among patients at a dermatology centre of Nghean province, Vietnam, 2015-2016. Mycopathologia 2017; 182 (11-12): 1061-1067

- Mirhendi H, Makimura K, de Hoog GS, Rezaei-Matehkolaei A, Javad Najafzadeh M, Umeda Y and Ahmadi B. Translation elongation factor 1- $\alpha$ gene as a potential taxonomic and identification marker in dermatophytes, Med Mycol 2015; 53 (3): $215-24$.
Correspondence address:

Silke Uhrlaß, Laboratory of Medical Microbiology, Moelbiser Hauptstrasse 8,

Roetha OT Moelbis 04571, Germany, E-Mail s.uhrlass@mykologie-experten.de www.mykologie-experten.de 